\title{
Sehnenabrisse am Becken
}

\author{
Stephan Lorenz, Andreas B. Imhoff
}

\section{Zusammenfassung}

Beckennahe Abrisse von Sehnen sind schwere Verletzungen, die unversorgt Funktionseinbußen zur Folge haben können. Aufgrund des ausgeprägten Weichteilmantels kann die Rupturlokalisation schwer eingeschätzt werden. Klinisch zeigt sich häufig ein Kraftverlust sowie ein Druckschmerz über der Ruptur bzw. der Insertion. Die bildgebende Diagnostik der Wahl ist das MRT sowie native Röntgenaufnahmen bei Verdacht auf ossäre Mitbeteiligung. Eine frühe operative Versorgung ist der konservativen Therapie und verspäteten Versorgung überlegen. Die Refixation erfolgt anatomisch über Fadenanker. Bei entsprechender Compliance ist eine Schienenversorgung häufig nicht nötig.

\section{Tendon Ruptures at the Pelvis}

Pelvic tendon ruptures are severe injuries that might lead to functional disability. Due to the large circumferential soft tissue the location and severity of the injury sometimes is hard to estimate. Clinical symptoms are muscle weakness and tenderness on palpation over the rupture and/or the insertion of the tendon. MRI is the preferred diagnostic imaging modality, whereas radiographies can detect concomitant osseous avulsions. Early surgical reinsertion with suture anchors is superior to conservative treatment or late reconstruction. Postoperative immobilization is rarely necessary in cooperative patients.

\section{Einleitung}

Sehnenabrisse am Becken sind eher seltene, aber schwerwiegende Verletzungen. Risikofaktoren für Rupturen sind der Verlauf der Muskeln über 2 Gelenke, rezidivierende Mikrotraumata, anaboles Doping, Einnahme/Injektion diverser Medikamente (z.B. Chinolone, Steroide) und verschiedene Stoffwechselerkrankungen (z.B. Diabetes).

Am häufigsten betroffen ist dabei die ischiokrurale Muskulatur (auch als „Hamstrings“ bezeichnet), die aus den Mm. semitendinosus, biceps femoris und semimembranosus besteht. Der Bizeps und Semitendinosus haben meistens eine gemeinsame Insertion am posterolateralen Tuber ischiadicum, der Semimembranosus unterschneidet die

OP-JOURNAL 2016; 32: 34-38

(C) Georg Thieme Verlag KG Stuttgart · New York DOI http://dx.doi.org/10.1055/s-0042-108596
Sehnen und inseriert anterolateral [1]. Als typischer Unfallmechanismus wird die forcierte Hüftflexion bei extendiertem Knie und gleichzeitiger Anspannung der Hamstring-Muskulatur angesehen wie z. B. beim verunglückten Wasserstart beim Wasserskifahren [2] oder dem Hürdenlaufen. Während es bei Adoleszenten eher $\mathrm{zu}$ einem knöchernen Abriss im Sinn einer Avulsionsfraktur kommt, sind bei Erwachsenen Rupturen im myotendinösen Übergang oder reine Sehnenabrisse häufiger [3].

Ein weiterer 2 Gelenke überspannender Muskel ist der $M$. rectus femoris der Quadrizepsmuskulatur. Der Muskel hat 2 Ursprünge. Die Pars recta inseriert an der Spina iliaca inferior, die Pars reflecta am Oberrand des Azetabulums. Beckennahe Ausrisse kommen hier v.a. beim Fußball in der Schussbewegung, z. B. bei einem Pressschlag, vor. Gefürchtet sind diese Aussrisse v.a. wegen einer früh einsetzenden Verkalkung, die in Flexion so störend ist, dass eine operative Entfer- nung der Verkalkungsspangen notwendig wird (Abb. 1).

Seltener ist der M. adductor longus mit seinem Ursprung am Ramus superior des Os pubis betroffen. Auch hier sind meist Fußballer bei Schussbewegungen betroffen.

Praktisch nur Kinder und Adoleszente sind von knöchernen Ausrissen der $\mathrm{Mm}$. sartorius und tensor fasciae latae an der Spina iliaca anterior superior betroffen. Ab einer Dislokation von mehr als $1 \mathrm{~cm}$ wird eine operative Insertion empfohlen (Abb. 2).

\section{Diagnostik}

\section{Klinische Untersuchung}

Anamnestisch berichten die Patienten meist über eine erhebliche Kraftanstrengung bzw. eine Bewegung über das normale Bewegungsausmaß hinaus, z. B. im Rahmen eines Sturzes. Plötzliche Schmerzen und Funktionseinschränkung mit Kraftverlust stehen unmittelbar nach Trauma im Vordergrund. In Abhängigkeit vom umgebenden Weichteilmantel kann es zu einem mehr oder weniger ausgeprägten Hämatom kommen. Häufig sinkt das Hämatom nach distal, sodass die eigentliche Rupturlokalisation übersehen wird. Es besteht Druckschmerz am Knochen und ggf. auch eine palpable Unterbrechung im Muskelgewebe.

Für Rupturen in der Hamstring-Gruppe ist eine Abschwächung der Flexion und der Außenrotation (Biceps femoris) und Innenrotation (Semitendinosus, Semimembranosus) in Kniebeugung pathognomonisch. Bei Rupturen des Rectus femoris ist häufig das Anheben des gestreckten Beines nicht mehr möglich, Schmerzen im Bereich des Schambeins sind für Läsionen des Adductor longus bei Anspreizung typisch. 


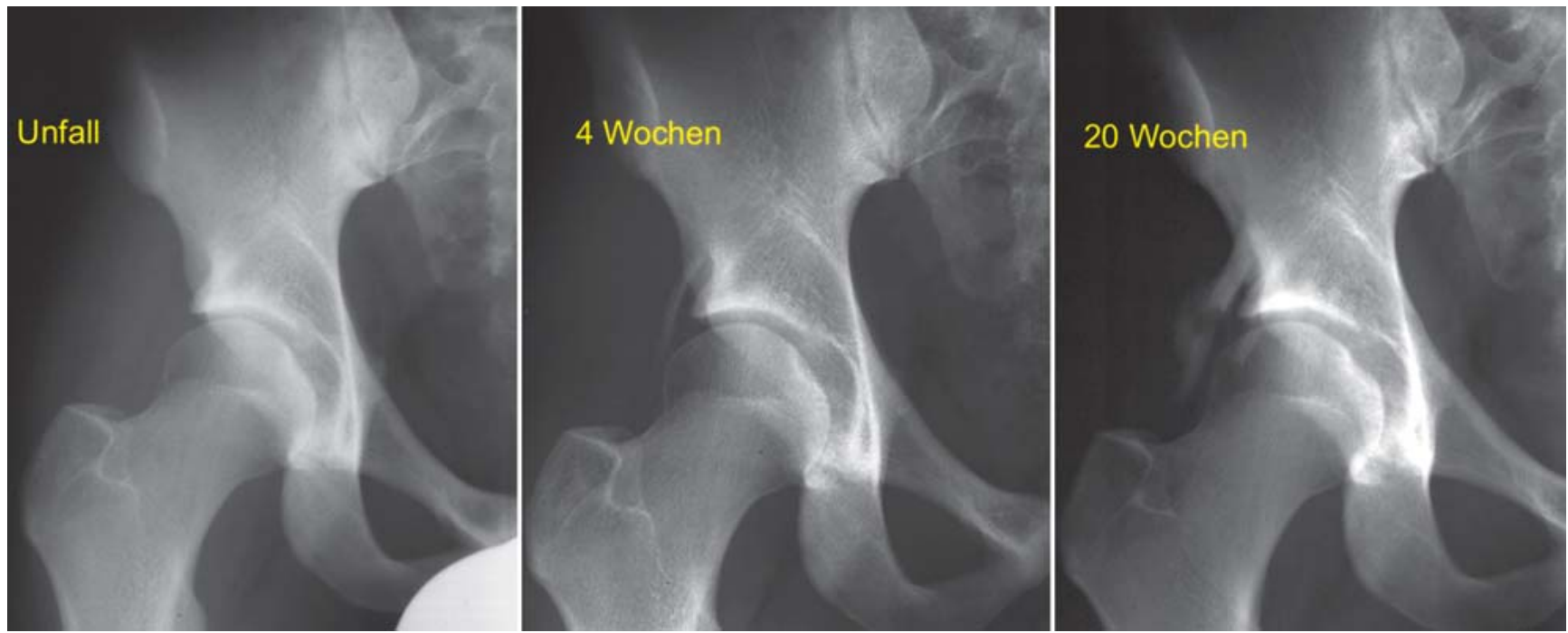

Abb. 1 Konservativer Verlauf eines M.-rectus-femoris-Abrisses. Initial keine ossäre Läsion sichtbar. Nach 4 Wochen Bildung einer feinen Ossifikationslinie (Mitte). Nach Sportkarenz und erneutem Pressschlag „Frakturierung“ der Knochenspange mit deutlicher Schmerzzunahme.

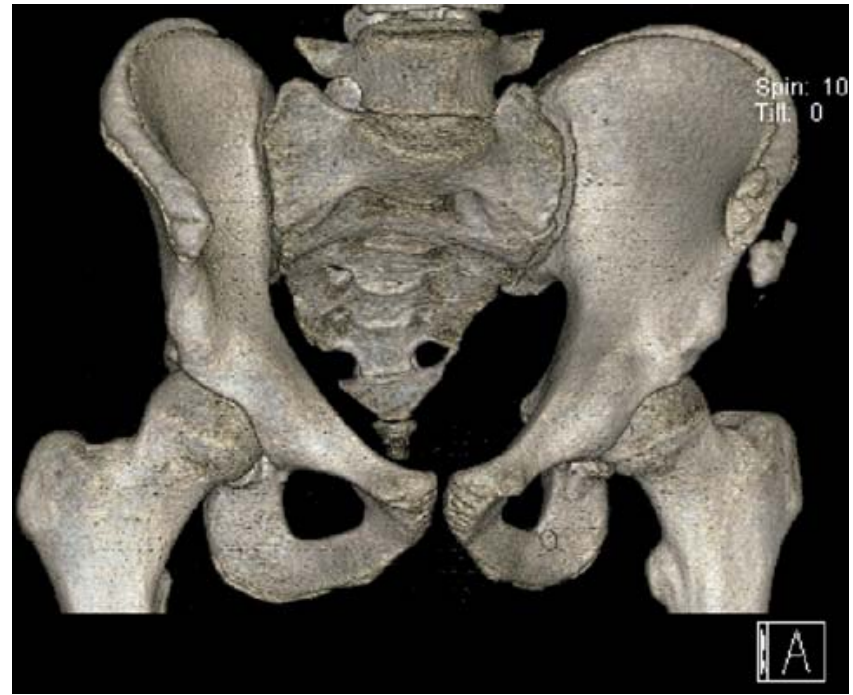

Abb. 2 Knöcherne Avulsion und Dislokation der Spina iliaca anterior superior links.

\section{Erweiterte Bildgebung}

Zum Ausschluss einer knöchernen Beteiligung sollten Röntgenbilder in 2 Ebenen evtl. ergänzt durch Spezialaufnahmen (inlet/outlet view) durchgeführt werden. Eine CT-Untersuchung ist nahezu immer entbehrlich und sollte aufgrund der hohen Strahlenbelastung der häufig jungen Patienten speziellen Fragestellungen (z.B. Ankerdislokation, Myositis ossificans) vorbehalten bleiben. Die Sonografie ist zur groben Orientierung sinnvoll, eine Magnetresonanztomografie ist aber die Standarduntersuchung zur Beurteilung von beckennahen Rupturen [4].

\section{Therapie}

\section{Operativ}

Beckennahe Komplettrupturen sollten heute in weitgehender Übereinstimmung mit der Literatur operativ versorgt werden. Dabei spielt der Zeitpunkt der OP eine übergeordnete Rolle. Die Rupturen sollten frühzeitig versorgt werden, eine Versorgung nach 3 Monaten hat eher schlechtere klinische Ergebnisse erbracht (Tab.1). Dies liegt nach eigener Erfahrung vor allem an den erheblichen Verwachsungen der abgerissenen Sehnen mit dem umgebenden Gewebe, die eine Mobilisierung erheblich erschweren oder unmöglich machen. Zudem ist das Risiko der Verletzung von neurovaskulären Strukturen deutlich erhöht.
Die Versorgung von Hamstring-Abrissen erfolgt in Bauchlage mit Unterpolsterung des Beckens und des Thoraxes. Eine Jodfolie schützt das OP-Gebiet zusätzlich vor Verunreinigung. Der Hautschnitt erfolgt bei mäßig- bis mittelgradiger Retraktion der Sehnen in der Glutealfalte (Abb. 3). Nach Durchtrennung der Körperfaszie, die nicht sehr ausgeprägt in diesem Bereich ist, erfolgt die stumpfe Präparation bis zum gut tastbaren Sitzbeinhöcker. Eine Präparation am Unterrand des $M$. gluteus maximus reduziert die Gefahr der temporären oder dauerhaften Schädigung der Äste des N. cutaneus femoris dorsalis, die auf der Faszie aufliegen. Nach lateral ist in der tiefen Präparation vor allem auf den N. ischiadicus zu achten, der im eigenen Vorgehen selten dargestellt wird, sondern 
Tab. 1 Literaturübersicht über frühzeitige und verzögerte operative Versorgung.

\begin{tabular}{|c|c|c|c|c|c|}
\hline Studie & Jahr & Design & $\mathbf{n}$ & Therapie & Ergebnis \\
\hline Wood D et al. [5] & 2008 & RCS & 72 & $\begin{array}{l}\text { Operation innerhalb von } 3 \text { Monaten bei } \\
\mathrm{n}=32\end{array}$ & $\begin{array}{l}\text { Sportfähigkeit nach } 6 \text { Monaten } \\
\text { bei } 80 \% \text {. Signifikant schlechtere } \\
\text { Ergebnisse bei verzögerter OP. }\end{array}$ \\
\hline Sarimo J et al. [6] & 2008 & RCS & 41 & $\begin{array}{l}\text { Operation innerhalb } 3 \text { Monaten bei } n=22 \text {, } \\
\text { nach } 3-6 \text { Monaten bei } n=12 \text { und nach } \\
\text { mehr als } 6 \text { Monaten bei } n=7\end{array}$ & $\begin{array}{l}\text { Odds ratio: } 29 \text {-faches Risiko für } \\
\text { schlechtes Ergebnis bei Opera- } \\
\text { tion nach mehr als } 3 \text { Monaten. }\end{array}$ \\
\hline Brucker P et al. [7] & 2004 & RCS & 8 & $\begin{array}{l}\text { operativ (innerhalb } 4 \text { Wochen post Trau- } \\
\text { ma bei } n=6 \text {, nach mehr als } 4 \text { Wochen } \\
\text { post Trauma bei } n=2 \text { ) }\end{array}$ & $\begin{array}{l}\text { Sehr gut in } 6 \text { von } 8 \text { Fällen mit } \\
\text { Sportfähigkeit auf Vorniveau } \\
\text { nach } 15 \text { Monaten. }\end{array}$ \\
\hline Klingele K et al. [8] & 2002 & RCS & 11 & $\begin{array}{l}\text { operativ (innerhalb } 4 \text { Wochen post Trau- } \\
\text { ma bei } n=7 \text {, nach mehr als } 4 \text { Wochen } \\
\text { post Trauma bei } n=4 \text { ) }\end{array}$ & $\begin{array}{l}\text { Sehr gut in } 10 \text { von } 11 \text { Fällen. } \\
\text { Sportfähigkeit nach } 6 \text { Monaten } \\
\text { bei } 7 \text { von } 9 \text { Fällen. }\end{array}$ \\
\hline Cross ] et al. [9] & 1998 & RCS & 9 & $\begin{array}{l}\text { operative Therapie nach durchschnittlich } \\
36(2-104) \text { Monaten }\end{array}$ & $\begin{array}{l}\text { Verbesserung in allen Fällen. } \\
\text { Sportfähigkeit bei } 7 \text { von } 9 \text { Fällen, } \\
\text { deutlicher Kraftverlust. }\end{array}$ \\
\hline Sallay P et al. [2] & 1996 & RCS & 12 & $\begin{array}{l}\text { primär konservativ, sekundär operativ bei } \\
n=2\end{array}$ & $\begin{array}{l}\text { Rekonvaleszenz } 3-18 \text { Monate. } \\
\text { Laufen nicht möglich bei } n=5 \text {. }\end{array}$ \\
\hline Orava S et al. [10] & 1995 & RCS & 8 & $\begin{array}{l}\text { operativ (innerhalb } 7 \text { Tagen post Trauma } \\
\text { bei } n=4 \text {, innerhalb } 3 \text { Monaten bei } n=2 \text {, } \\
\text { innerhalb } 18 \text { Monaten bei } n=2 \text { ) }\end{array}$ & $\begin{array}{l}\text { Nach Früh-OP gut, nach Spät-OP } \\
\text { mäßig. }\end{array}$ \\
\hline
\end{tabular}

RCS: randomisiert kontrollierte Studie

im Weichteilverbund nach lateral gehalten wird. In den meisten Fällen ist die Muskelfaszie der Hamstrings noch erhalten und muss knochennah durchtrennt werden. Es entleert sich altes Hämatom, die Sehnenstümpfe sind in diesem Bereich gut zu tasten und werden stumpf mobilisiert, bis sie spannungsfrei an den Knochen zurückgeführt werden können. Es erfolgt die Anfrischung des freiliegenden Knochens am Sitzbeinhöcker mit dem scharfen Löffel. Setzen von meist 2 Fadenankern CorkScrewFT 5,5 mm (Arthrex, Freiham) nach Vorbohren mit dem 3,5-mm-Bohrer. Im eigenen Kollektiv werden Titananker zur besseren postoperativen Evaluation (Abb.4) und höheren Ausrisskraft verwendet. Biomechanische Studien konnten zeigen, das die Verwendung von 5 Ankern eine größere primäre Ausrisskraft bewirkt [11], in der eigenen Gruppe ist eine Versorgung mit weniger Ankern aber für die Einheilung ausreichend. Die Sehnenplatte wird mit jeweils einem Fadenende mit überschlagenden Nähten angeschlungen und über das 2. Fadenende an den Knochen gezogen und verknotet. Der oberflächliche Wundverschluss erfolgt nach Einlage einer nach lateral ausgeleiteten Redon-Drainage. Die Nachbehandlung erfolgt bei spannungsreicher Re-Insertion für 6 Wochen mithilfe einer Newport-Orthese, die die Beugung in der Hüfte limitiert. Bei starker Spannung kann mit einem Fußeinschluss auch die Streckung im Knie limi-

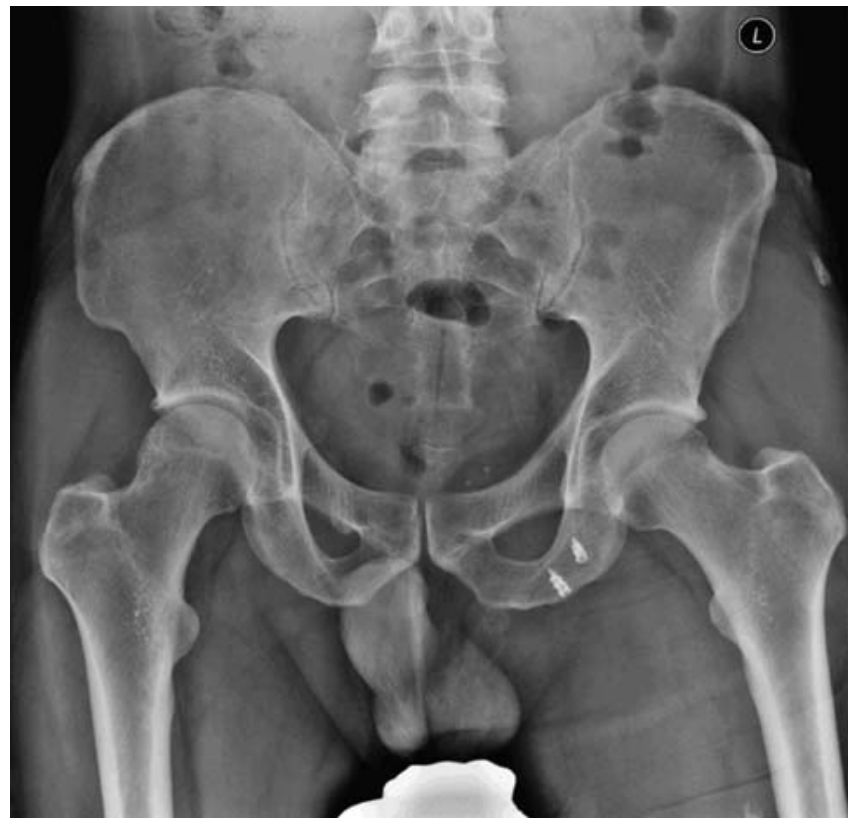

Abb. 4 Postoperative Kontrolle der Ankerlage. tiert werden. Bei frühzeitiger Versorgung lässt sich aber unter Teilbelastung $20 \mathrm{~kg}$ und Schulung des Patienten (Beugung der Hüfte nur gleichzeitig mit Beugung des Knies) eine sehr störende Schienenversorgung vermeiden. Die klinischen Ergebnisse sind durchweg gut mit nur leichtem Kraftverlust und Rückkehr zum alten Sportaktivitätsniveau nach 2 Jahren [7]. Auch Partialrupturen sollten nach einem unbefriedigenden konservativen Therapieversuch operiert werden und können mit guten Ergebnissen zum Sport zurückkehren [12].

Abrisse des M. rectus femoris werden in Rückenlage über einen geraden Zugang von der Spina iliaca anterior superior nach distal operiert (Abb. 5). In der weiteren Präparation wird der N. cutaneus femoris lateralis identifiziert, der über der Faszie des M. sartorius liegt (Abb. 6). Die Faszie wird zwischen dem M. sartorius und M. tensor fasciae latae inzidiert 


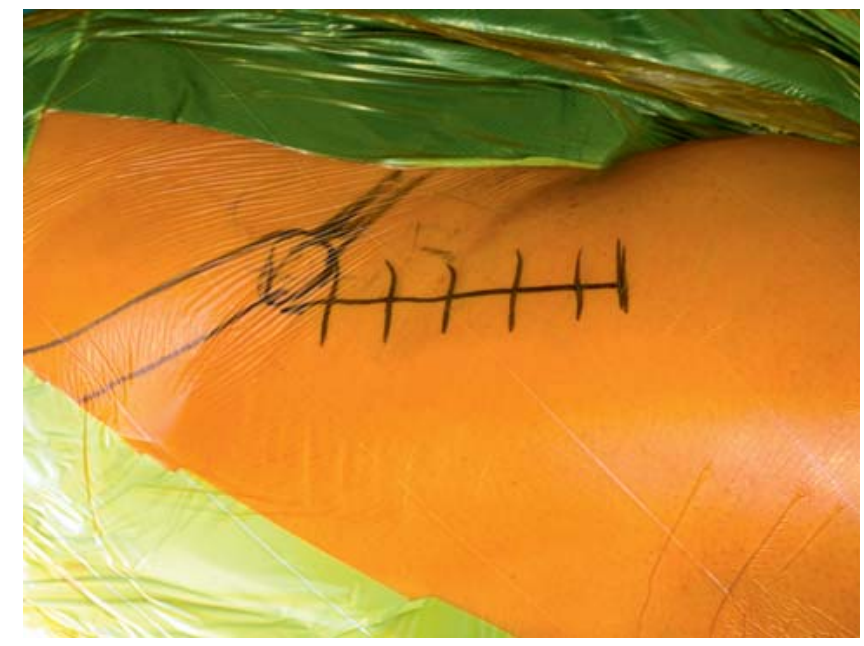

Abb. 5 Geplanter Hautschnitt für Refixation des M. rectus femoris ca. $7 \mathrm{~cm}$ von der Spina iliaca anterior superior nach distal.
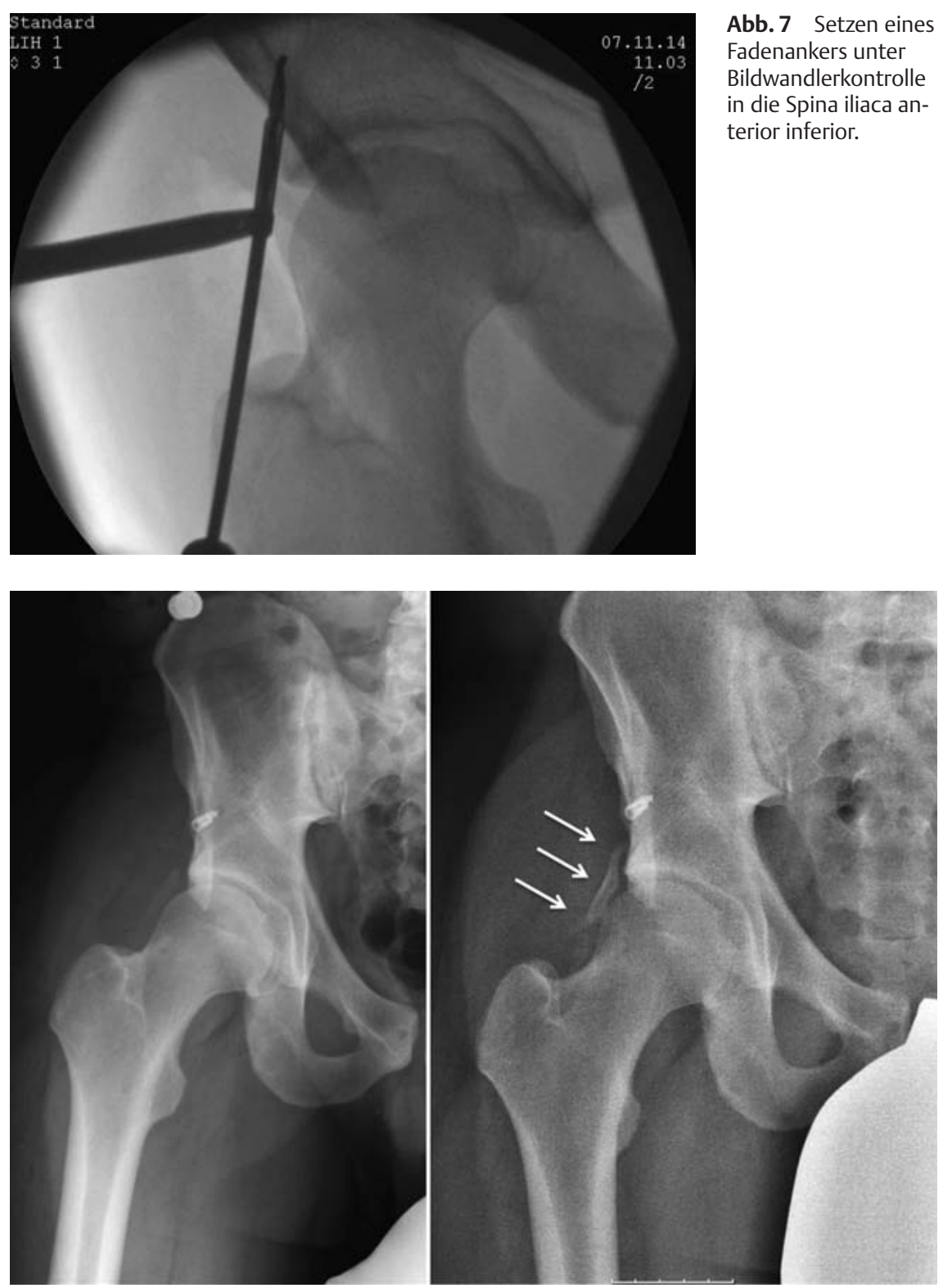

Abb. 8 Röntgenkontrolle 2 Tage (links) und 6 Wochen (rechts) post OP. Entwicklung einer Ossifikation (Pfeile) nach Rekonstruktion des M. rectus femoris mit Fadenanker.
Abb. 7 Setzen eines Fadenankers unter Bildwandlerkontrolle in die Spina iliaca anterior inferior.

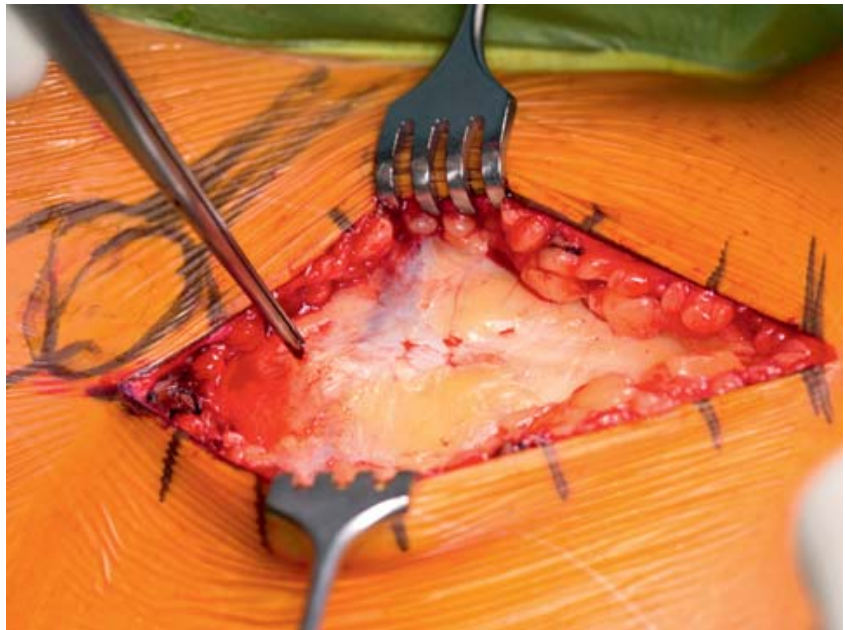

Abb. 6 Identifikation des N. cutaneus femoris lateralis (Pinzette) und Inzision der Faszie zwischen M. tensor fasciae latae und M. sartorius.

und die Muskeln stumpf auseinander gedrängt. Darunter kommt der $\mathrm{M}$. rectus femoris zu liegen, der mit seiner relativ kurzen Sehnenplatte bis zum Ansatz verfolgt wird. Meistens ist der Ansatz an der Spina iliaca anterior inferior betroffen. Mithilfe des Bildwandlers wird ein Fadenanker gesetzt (Abb. 7) und der Sehnenstumpf angeschlungen und fixiert. Die Nachbehandlung erfolgt unter Teilbelastung $20 \mathrm{~kg}$ und Limitierung der Hüftbeugung (Flex/Ex frei-20-0) für 6 Wochen meist ohne Newport-Orthese. Eine Ossifikationsprophylaxe sollte unbedingt eingenommen werden, da die Sehne zu Verkalkungen neigt (Abb. 8).

Der Adductor longus wird in Rückenlage unter Abspreizung des betroffenen Beines operiert. Der Hautschnitt erfolgt dabei in der Inguinalfalte (Abb.9). Besonderes Augenmerk muss in der Präparation auf die Schonung des Funiculus spermaticus, der bei Männern lateral des Hautschnitts verläuft, gelegt werden. Die Fadenanker werden unter Durchleuchtung vorgebohrt und eingebracht. Die Symphyse darf dabei nicht affektiert werden (Abb. 10). Eine Orthesenbehandlung ist normalerweise nicht notwendig. Abduktion und Flexion über $60^{\circ}$ sollen für 6 Wochen vermieden werden.

Die Refixierung der Spina iliaca anterior superior mit den Ansätzen der Mm. tensor fasciae latae und sartorius sowie dem Inguinalband erfolgt über einen Zugang direkt über der Spina. Der medial davon gelegene $\mathrm{N}$. cutaneus femoris lateralis muss dabei geschont werden. Die Spina wird in einer Cerclage-Technik gefasst und re-inseriert. Dabei werden 2 mit FiberWire armierte BioComposit-SwiveLock-4,75-mm-Anker am inferioren 


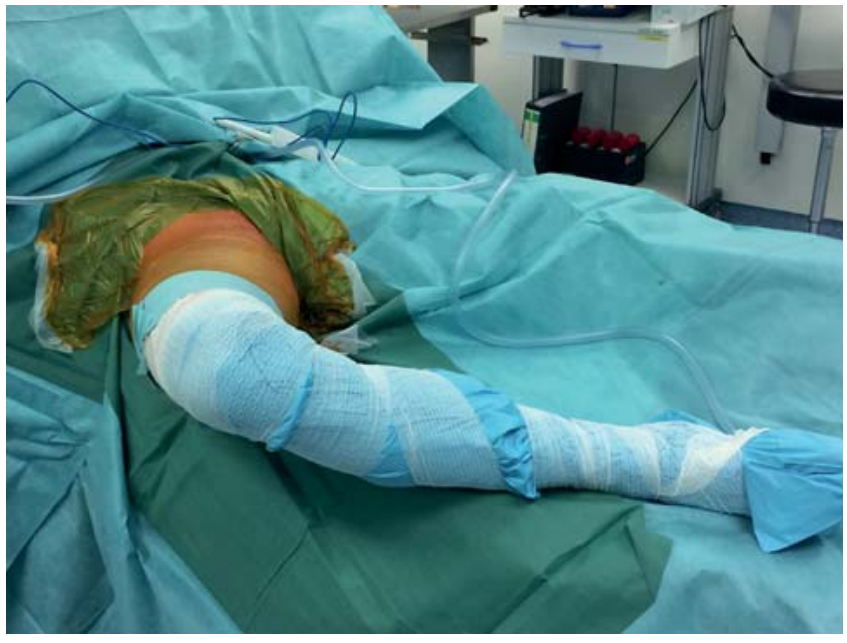

Abb.9 Lagerung für M.-adductor-longus-Refixation.

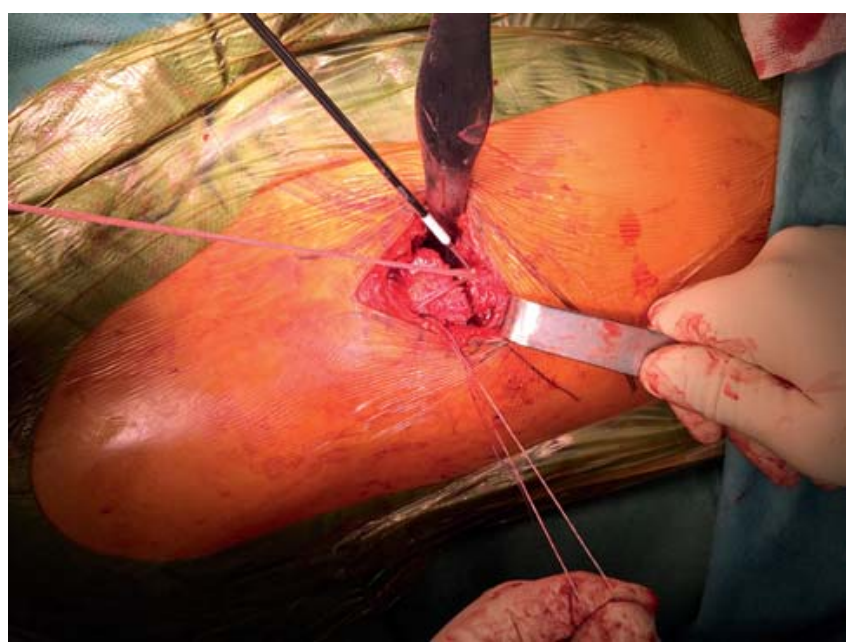

Abb. 11 Setzen de 1. PushLock-Ankers und Verspannen der FiberWire-Fäden in Cerclage-Technik. Die Fäden der SwiveLockAnker wurden bereits durch die Sehnen distal des Fragments durchgestochen.

Rand der Insertion eingedreht. Die Sehnen werden mit den Fäden durchstochen und über das Spinafragment geführt. Die Spannung und Fixation der Fäden erfolgt durch 2 PushLock-Anker an der Crista iliaca (Abb. 11). Unter Ossifikationsprophylaxe mit NSARs erfolgt die Teilbelastung für 6 Wochen.

\section{Literatur}

${ }^{1}$ Feucht MJ, Plath JE, Seppel G et al. Gross anatomical and dimensional characteristics of the proximal hamstring origin. Knee Surg Sports Traumatol Arthrosc 2014; 23: 25762582

2 Sallay PI, Friedman RL, Coogan PG et al. Hamstring muscle injuries among water skiers Functional outcome and prevention. Am J Sports Med 1996; 24: 130-136
${ }^{3}$ Miller SL, Gill J, Webb GR. The proximal origin of the hamstrings and surrounding anatomy encountered during repair. A cadaveric study. J Bone Joint Surg Am 2007; 89: 44-48

4 Kerkhoffs GMMJ, van Es N, Wieldraaijer T et al. Diagnosis and prognosis of acute hamstring injuries in athletes. Knee Surg Sports Traumatol Arthrosc 2012; 21: 500-509

${ }^{5}$ Wood DG, Packham I, Trikha SP et al. Avulsion of the proximal hamstring origin. J Bone Joint Surg Am 2008; 90: 2365-2374

${ }^{6}$ Sarimo J, Lempainen L, Mattila K et al. Complete proximal hamstring avulsions: a series of 41 patients with operative treatment. Am J Sports Med 2008; 36: 1110-1115

7 Brucker PU, Imhoff AB. Functional assessment after acute and chronic complete ruptures of the proximal hamstring tendons. Knee Surg Sports Traumatol Arthrosc 2004; 13: 411418

8 Klingele KE, Sallay PI. Surgical repair of complete proximal hamstring tendon rupture. Am J Sports Med 2002; 30: 742-747
${ }^{9}$ Cross MJ, Vandersluis R, Wood D et al. Surgical repair of chronic complete hamstring tendon rupture in the adult patient. Am J Sports Med 1998; 26: 785-788

10 Orava S, Kujala UM. Rupture of the ischial origin of the hamstring muscles. Am J Sports Med 1995; 23: 702-705

${ }^{11}$ Hamming MG, Philippon MJ, Rasmussen MT et al. Structural properties of the intact proximal hamstring origin and evaluation of varying avulsion repair techniques: an in vitro biomechanical analysis. Am J Sports Med 2015; 43: 721-728

12 Bowman KF, Cohen SB, Bradley JP. Operative management of partial-thickness tears of the proximal hamstring muscles in athletes. Am J Sports Med 2013; 41: 1363-1371

\section{PD Dr. med. Stephan Lorenz} Chefarzt

Abteilung Sportorthopädie Chirurgisches Klinikum München Süd Am Isarkanal 30 81379 München

stephan.lorenz@artemed.de

Univ.-Prof. Dr. med. Andreas B. Imhoff Chefarzt

Abteilung Sportorthopädie Klinikum rechts der Isar Technische Universität München Ismaninger Straße 22 81675 München

a.imhoff@lrz.tum.de 